\title{
Challenges in Very High Resolution Imaging of Satellites and Objects in Space
}

\author{
M. Jirousek, S. Anger, S. Dill, M. Peichl \\ DLR German Aerospace Center, \\ Microwaves and Radar Institute, \\ Department of Reconnaissance and Security, \\ Muenchener Str. 20, 82230 Wessling, Germany
}

\begin{abstract}
Space debris nowadays is one of the main threats for satellite systems especially in low earth orbit (LEO). More than 700,000 debris objects with potential to destroy or damage a satellite are estimated. The effects of an impact often are not identifiable directly from ground. High-resolution radar images are helpful in analyzing a possible damage. Furthermore investigations on unknown space objects or satellites can be performed. Therefor DLR is currently developing a radar system called IoSiS (Imaging of Satellites in Space), being based on an existing steering antenna structure and our multipurpose high-performance radar system GigaRad for experimental investigations. GigaRad is a multi-channel system operating at $\mathrm{X}$ band and using a bandwidth of up to $4.4 \mathrm{GHz}$ in the IoSiS configuration, providing fully separated transmit (TX) and receive (RX) channels, and separated antennas. For the observation of small satellites or space debris a high-power traveling-wave-tube amplifier (TWTA) is mounted close to the TX antenna feed. For the experimental phase IoSiS uses a $9 \mathrm{~m}$ TX and a $1.8 \mathrm{~m} \mathrm{RX}$ antenna mounted on a common steerable positioner. High-resolution radar images are obtained by using Inverse Synthetic Aperture Radar (ISAR) techniques. The guided tracking of known objects during orbit pass allows here wide azimuth observation angles. Thus high azimuth resolution comparable to the range resolution can be achieved. This paper outlines technical main characteristics of the IoSiS radar system. It shows the main error sources and solutions as well as the calibration effort to generate the first centimeter-resolution radar image observed with IoSIS.
\end{abstract}

Keywords: space surveillance, inverse synthetic aperture radar, ISAR, high-resolution radar, space debris, space system situational awareness

\section{INTRODUCTION}

Since the first satellite Sputnik 1 has been launched in October 1957 the number of satellites in orbit has increased to almost 2000 operational and 3000 out-of-service ones, according to ESA's database. Hereby the trend is more exponential than linear. Especially NewSpace, a movement and philosophy encompassing a globally private spaceflight industry of new aerospace companies and ventures working independently of governments and traditional major contractors, multiply the amount of satellites and launches. Until the end of 2018 over 1000 Nano and CubeSats were launched. In the next 6 years more than 3000 new Nanosats are planned for launch. This illustrates the significance of space not only for research and reconnaissance purposes but also as a growing economic region. This is even more visible in the amount of countries operating satellites and the numbers of countries having a space launching capability. The global distribution of these countries is shown in the Figure 1 for the year 2016 [10].

This leads to three main problems mostly in the low earth orbit (LEO): a tremendous increase of space debris, the potential hazard of damage and destruction by space debris and unknown objects. For detection of debris a few systems are operating in the optical and radar domain worldwide, for instance TIRA form FHR [13], RAF Fylingdales from Royal Air Force [11], United States BMEWS network [12]. To analyze the impact of debris on an operating satellite or to classify and identify an unknown tracked object a high-resolution imaging capability is essential. The required spatial resolution for these needs is in the centimeter range. To provide this resolution considering optical imaging of objects in the LEO, telescopes with a diameter of more than ten meter and an enormous effort in atmospheric correction is required. Considering a radar instrument the same resolution with a similar antenna diameter, compared to the optical telescope, but a way less requirements and atmospheric corrections are needed and in addition an all day and weather 
capability is achieved. This was the reason to build up an experimental radar instrument called IoSiS (Imaging of Satellites in Space) to study the challenges for an operational very high resolution imaging system for future issues, being not adequately addressable by today's classic imaging approaches.

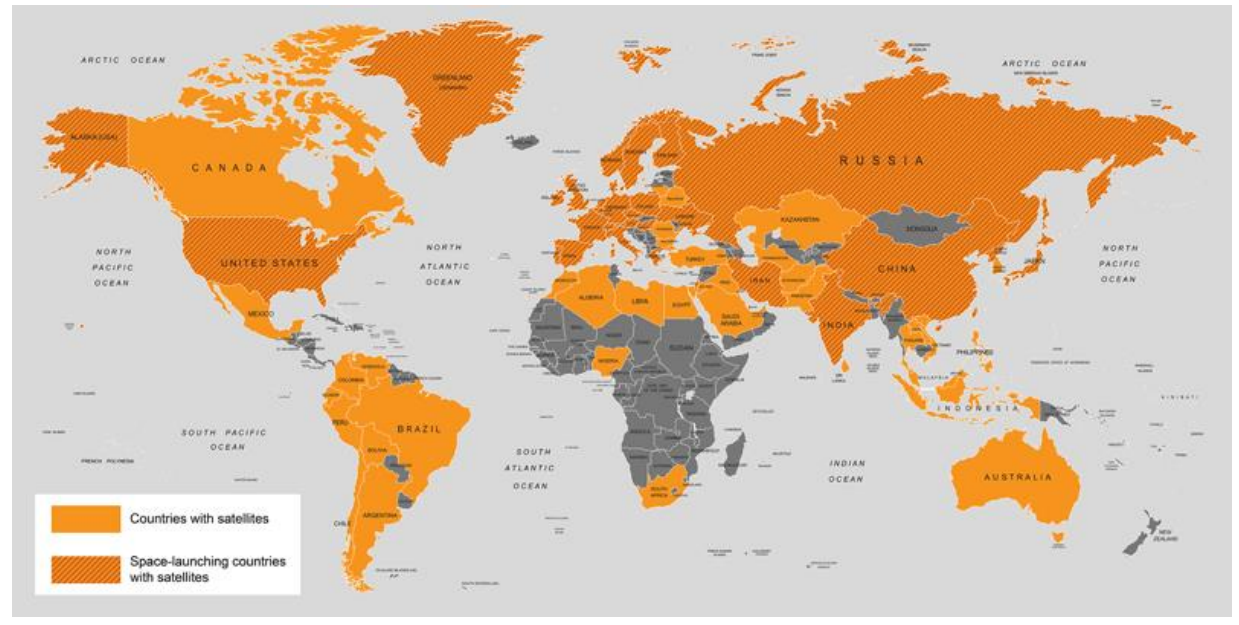

Figure 1. Worldwide countries with satellites and space-launching capabilities 2016 (Union of Concerned Scientists)

\section{IOSIS SYSTEM DESIGN}

The experimental system IoSiS is setup at the DLR ground station in Weilheim, close to Munich, were the commanding of a variety of satellites is performed with several antennas (Figure 2). To save cost and bureaucratic obstacle IoSiS uses an existing steerable communication antenna which was used as a backup for $\mathrm{S}$ band commanding. The 50 years old antenna was redesigned to a Cassegrain broadband $\mathrm{X}$ band antenna with a high-power feeding in the back shown in Figure 3.

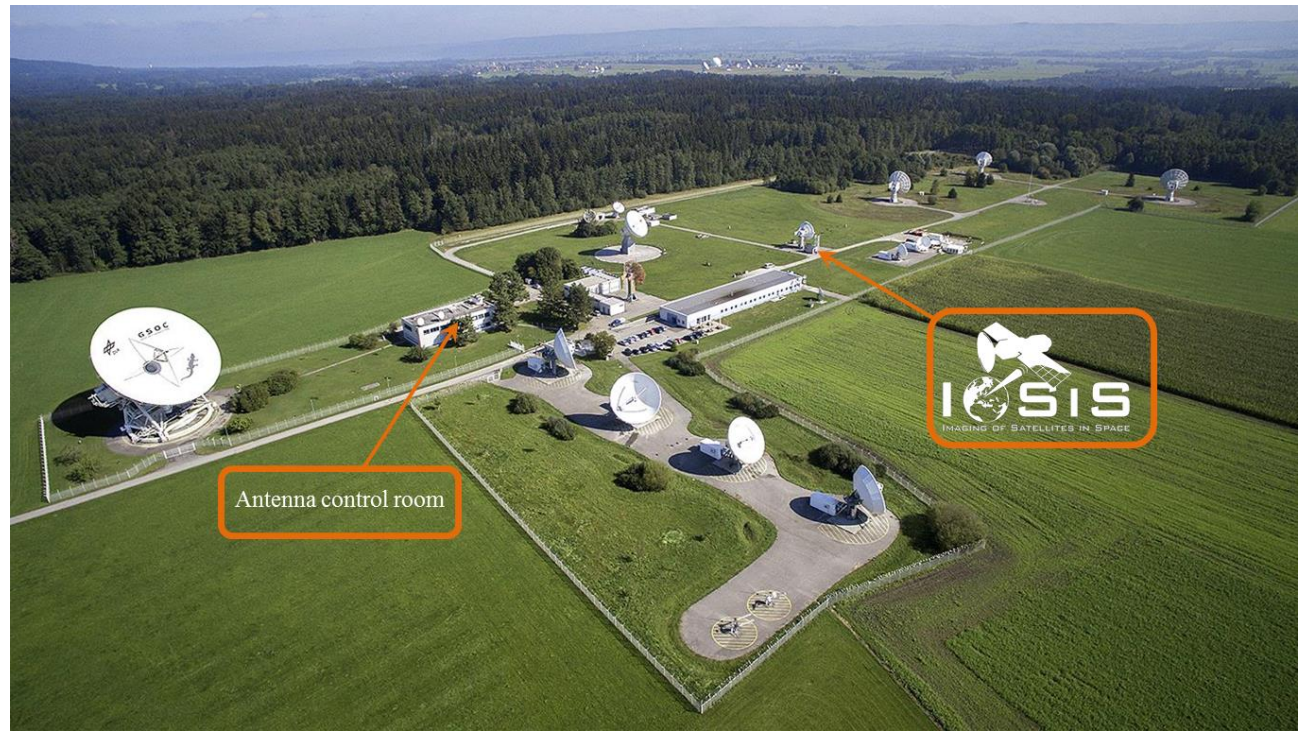

Figure 2. Aerial image of IoSiS location at the DLR ground station in Weilheim with the antenna control room.

Two $1.8 \mathrm{~m}$ receive $(\mathrm{Rx})$ antennas are flanged beside the existing $9 \mathrm{~m}$ antenna on both sides to allow a two channel receive operation for future imaging modes, like polarimetric or interferometric measurements. The high power amplifier is located as close as possible in the back of the antenna. The main radar electronic and control is housed in a container nearby the positioner base. Here the whole instrument configuration and programming as well as the image acquisition are conducted. The radar instrument called GigaRad [1] used for this purpose is shown in Figure 4. 


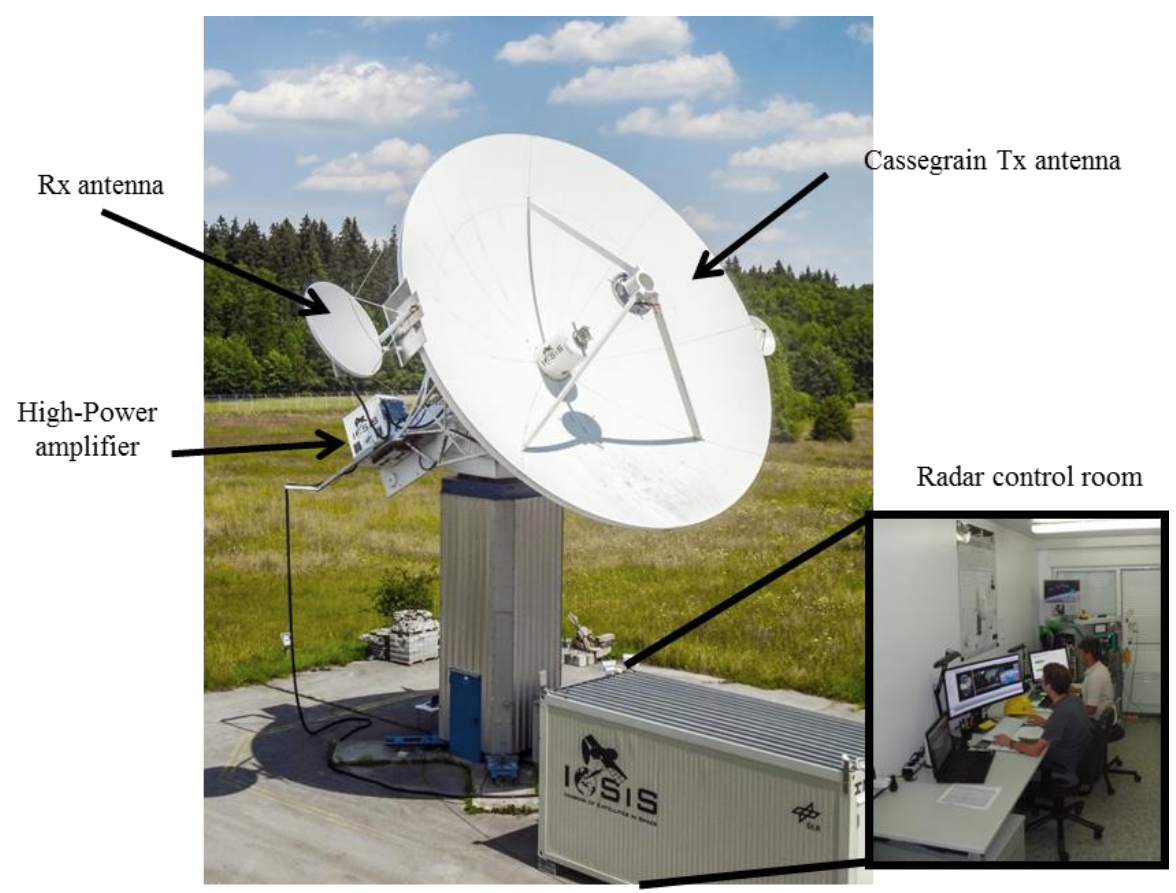

Figure 3. IoSiS System setup. Two small Rx-antennas are mounted at the reflector of the steerable Tx-antenna. The high power amplifier and distribution electric is located in the back of the antenna. A nearby container showing the radar control room.

GigaRad is a very flexible $\mathrm{X} / \mathrm{Ku}$-band pulse radar with a digital signal generation and a sampling in real-time with up to $6 \mathrm{GHz}$ bandwidth. In the IoSiS configuration in Weilheim the bandwidth is restricted to $2.8 \mathrm{GHz}$ due to interference limitation to other services close by. The main operational parameters are a pulse repetition frequency of $200 \mathrm{~Hz}$, a linear frequency modulated pulse with up to $50 \mu$ s length, a center frequency of $9.4 \mathrm{GHz}$ and an output power of $8 \mathrm{~kW}$.

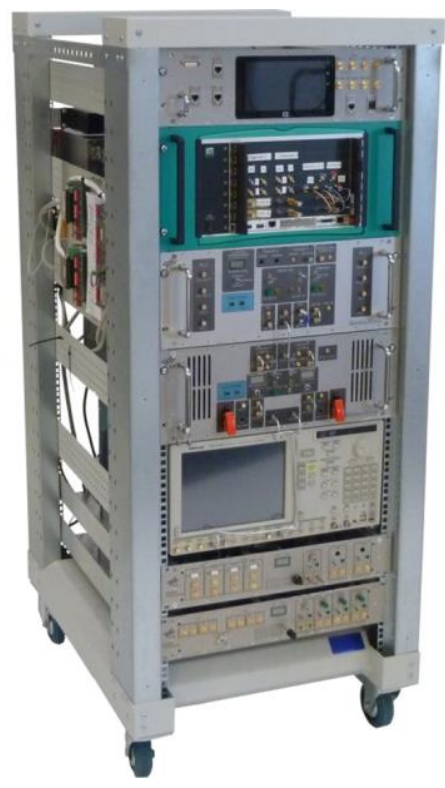

Digital control unit

Analog-to-digital converter

Rx signal conditioning module

Tx signal conditioning module

Arbitrary waveform generator

Tx and Tx extension modules

Figure 4. Photograph of the basic radar hardware setup of GigaRad including the extension modules, all being installed in a mobile 19"' rack. 
The bandwidth of $2.8 \mathrm{GHz}$ corresponds to a theoretical resolution of $5.3 \mathrm{~cm}$ in range. To acquire a two dimensional image with an equal resolution in the second dimension, in azimuth, IoSiS uses the inverse synthetic aperture principal [4] as shown in Figure 5. The steerable antenna is thereby programmed to follow a space object. During the orbit pass the radar collects range profiles processed in further steps, described in detail in section 3.4, to an ISAR image. Hereby the angle corresponds to the synthetic aperture length, needed for ISAR processing. For equal spatial resolution in both image directions the angle should be around $17^{\circ}$.

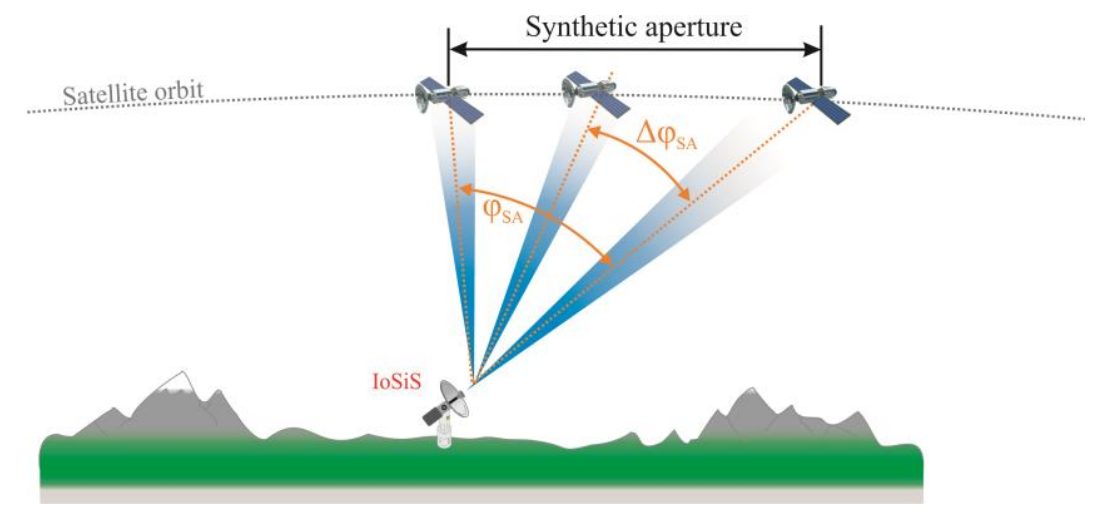

Figure 5. IoSiS satellite imaging geometry with a steerable antenna following the satellite on its orbit path in order to acquire range profiles over a large synthetic aperture path for ISAR processing.

\section{IOSIS CHALLENGES}

The functionality of IoSiS to image objects in a LEO offers some advantages and some disadvantages compared to an orbiting remote sensing radar satellite. On the one hand the radar is stationary and accessible which gives much more flexibility. The image area of interest is an object in an empty space without backround clutter. Hence no other device can disturb due to ambiguities, caused for example by an inadequate sampling or a less side lope suppression of the antenna. On the other hand it is challenging to find an object in an empty space with no orientation in the data. Also the reflected energy is way less for small objects in space than for a whole scenario on ground. Another important point is the required spatial resolution which is minimum a factor of 5 higher than satellite missions with the best known resolution today. This leads to huge challenges which are addressed in this chapter.

\subsection{Instrument design}

The key factor for a very high resolution image is a specific accurate instrument design. Hence, IoSiS is an experimental system, existing of easy to access devices. As basic radar system the multi-purpose advanced X band radar GigaRad is used [1]. It is based on a purely digital signal generation with an arbitrary waveform generator. This enables much functionality in generating waveforms and signal predistortion. A one step heterodyne concept converts the signal to X band where it is filtered, amplified and transferred via optical link to the high power amplifier (HPA) in the back of the Cassegrain antenna system of $9 \mathrm{~m}$ diameter for transmit (TX) [7]. The closest positioning of the HPA to the feed antenna is a very important point to have the maximum output power available and a minimum phase and amplitude distortion for instance in a slip-ring. For a maximum signal-to-noise ration (SNR) the output power should be sufficient high and should provide a flat frequency response. Beside the predistortion in amplitude and phase, equalization with a filter guaranties best results. IoSiS achieves almost flat response with a peak power of $6 \mathrm{~kW}$ over the whole bandwidth at the feed antenna.

Because of wind-load requirements and the intention to investigate for future systems the Rx-antenna gain is limited. So, as SNR is the main driver for observation of objects with little RCS (e.g. smaller satellites) or objects in a higher orbit in a larger distance, a careful attention is payed on the Rx-side to the low-noise-amplifier. The noise figure of the amplifier and the position close to the feed are well designed. Also the attenuation as well as the matches of the connections is minimized. The physical separation of Tx and Rx paths also provides sufficiently high isolation taking into account the large bandwidth and the high pulse power. The Rx signal is amplified, filtered, transferred via optical link, adjusted and down converted like in the Tx path. For maximum flexibility the signal is sampled in the time-domain with a sample rate of $8 \mathrm{GS} / \mathrm{s}$. In combination with a proper calibration strategy, which is described in the next section, a correction of the signals is possible. 
The analysis of a radar signal and especially the processing of a SAR image require a coherent system. The main requirement for coherency is the phase stability and phase noise as well as jitter. Therefore the GigaRad instrument has a core reference oscillator and frequency-generation unit. The reference oscillator is a low noise Rubidium GlobalNavigation-Satellites-System Disciplined Oscillator (GNSSDO) with a highest performance available. The low phase noise Frequency Generation unit (FGEN) concentrates all signal sources and links them to the GNSSDO. A careful distribution of the signals to the mixers, the ADC and the DAC guaranties a minimum distortion of the image.

Because of the experimental status of IoSiS no link to a tracking system for objects in the LEO is realized. For observation of the objects the radar instrument includes a decent timing unit to program the correct distance for the receive window at the correct time. The GigaRad instrument uses a field programmable gate array (FPGA) chip with GNSS timing. The clock of the FPGA is also generated in the FGEN and therefor synchronized to all other components of the radar. The GNSS timing is based on the Pulse-Per-Second (PPS) signal with accuracy better than $100 \mathrm{~ns}$ which is sufficient for trigger signals.

\subsection{Calibration}

IoSiS is based on pulse radar with mainly linear frequency modulation. The theoretical ideal impulse response of such a signal is a squared $\sin (\mathrm{x}) / \mathrm{x}$ function. The very high bandwidth combined with the output power results in imperfections and error sources. In chapter 3.1 many aspects are considered to reduce these errors. The remaining effects are almost eliminated with an adapted calibration strategy. For IoSiS three main calibration steps are performed [7]. First a calibration loop that guides the Tx signal to the Rx path measures the frequency response function with respect to phase, group delay and amplitude. In this step all components except the waveguide to the Tx feed antenna and the antennas itself on Tx and Rx side are measured. An external calibration with a trihedral reflector characterizes these components in a second step and links the measured values to an absolute RCS. To reduce the impact of drifts, before and after each measurement an inner calibration measurement is performed. Only with these steps a sufficient compression of the pulses in range is achieved for the desired image resolution of a few centimeters.

\subsection{Precise orbit prediction}

In order to illuminate the satellite over a sufficient long time and azimuth angle, respectively, the steerable antenna system has to follow precisely the satellite on its orbit path during the pass. To ensure, that this constant illumination is maintained, the orbit path has to be known very precisely during the satellite path. Otherwise the satellite isn't within the antenna main beam and range profiles can't be acquired. Because IoSiS doesn't have an auto tracking capability so far, there is the need to predict the path out of public available Two-Line-Element datasets (TLE). This prediction is defective with a specific margin of error due to atmospheric drag and the aspherical gravitational field due to, among others, the oblateness of the earth at the poles.

The prediction error shows Figure 6 for instance for the satellite Terra-SAR-X with an orbital height of $514 \mathrm{~km}$. Depicted is the position difference between the real satellite position of TerraSAR-X, determined based on GPS data [5], and the orbit path predicted based on TLE. Here the position error is split in three parts. The "Cross" and the "Radial" error components are in the region of about a few hundred meters, whereby the error in flight direction increases up to a maxim value of about $6 \mathrm{~km}$. If we consider the orbital velocity the maximum position error corresponds to a time offset of about $0.79 \mathrm{~s}$. For IoSiS the antenna half-power beam width (HPBW) is about $0.2^{\circ}$. This corresponds to an antenna footprint of about $2.7 \mathrm{~km}$ with the result that for some orbit paths the object is not visible. One solution to overcome this problem is prior to determine the time offset by using two subsequent orbits. The first pass is used to determine the time offset using a fixed aligned antenna and the second one for the actual imaging measurement[8].

Another challenge to an experimental system is the antenna positioning system. As described an adaption from an $\mathrm{S}$ band communication system to $\mathrm{X}$ band was carried out. Even though the 50 years old positioner is equipped with a newer Antenna Control Unit (ACU), the whole positioner has been designed for an accuracy 5 times less than necessary for an Xband system yielding to a misalignement. Considering an observation of an object with precisely known orbit data, this misalignment of the antenna beam will lead to a loss of SNR, because if the antenna beam points not directly in the direction of the space object the antenna gain is reduced. In case the offset is too high no image reconstruction is possible any more. To verify the movement of the antenna, a test setup with two optical cameras has been installed. One camera is adapted to the sub-reflector of the Tx-antenna and one on the top of one of the Rx-antennas as shown in Figure 7. For a normal operational path the antenna was programmed to follow the predicted orbit of the ISS. In twilight conditions, meaning that at the observation point on ground is sufficient dark, whereas in orbit the object is still illuminated by the sun, the ISS is visible by human eyes or an optical camera. If the pointing of the camera is aligned properly with the 
antenna pointing the reflected light is visible as a bolt in the image. For ideal antenna movement the bolt would stay at the same position/pixel for the whole pass. As shown in Figure 8 the bolt stays not centered. If considering the Tx HPBW as the red boundary the ISS is out of the HPBW. The two examples are for the nearest approach where the azimuth rotation of the antenna is highest and the miss-pointing is highest.

The result for the experimental IoSiS setup is that even for an absolute precise prediction IoSiS could lose performance. Hence one challenge is a precise orbit prediction based on actual state vectors and an accurate positioning system or a predistortion of the current unprecise positioner to eliminate the deviation.

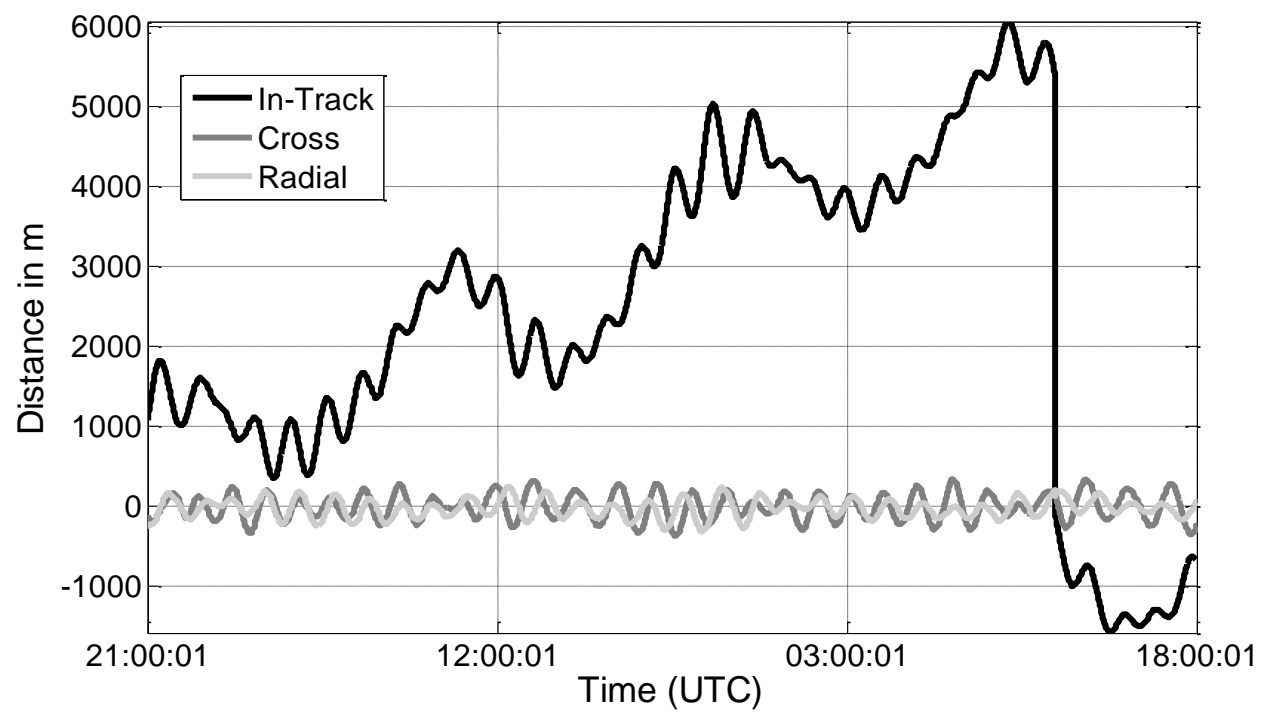

Figure 6. Comparison between the real satellite position of TerraSAR-X, determined based on GPS data [5], and the orbit path predicted based on Two-Line-Elements (TLE).

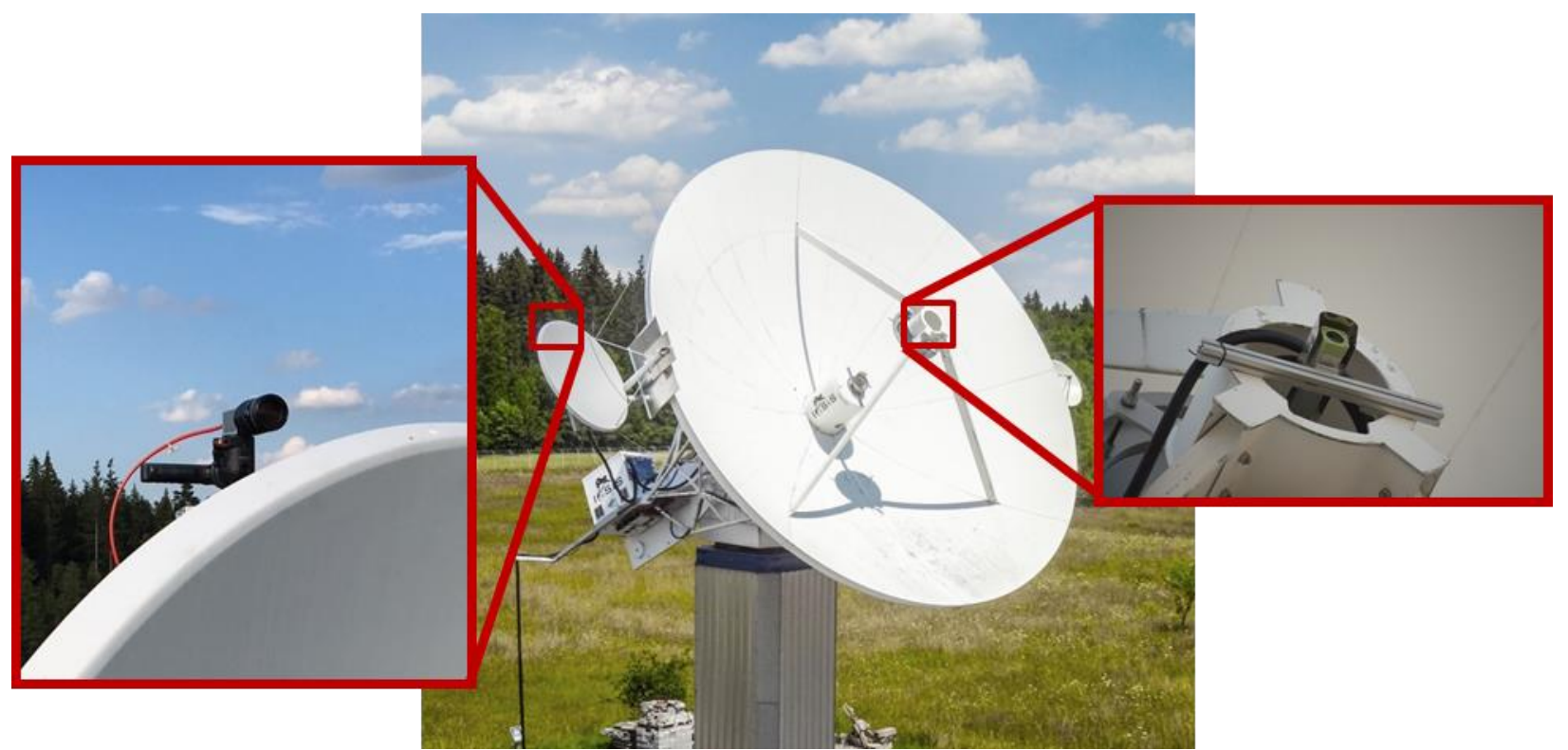

Figure 7. Assembly of a test setup capturing the ISS with two optical cameras to verify the antenna motion. 

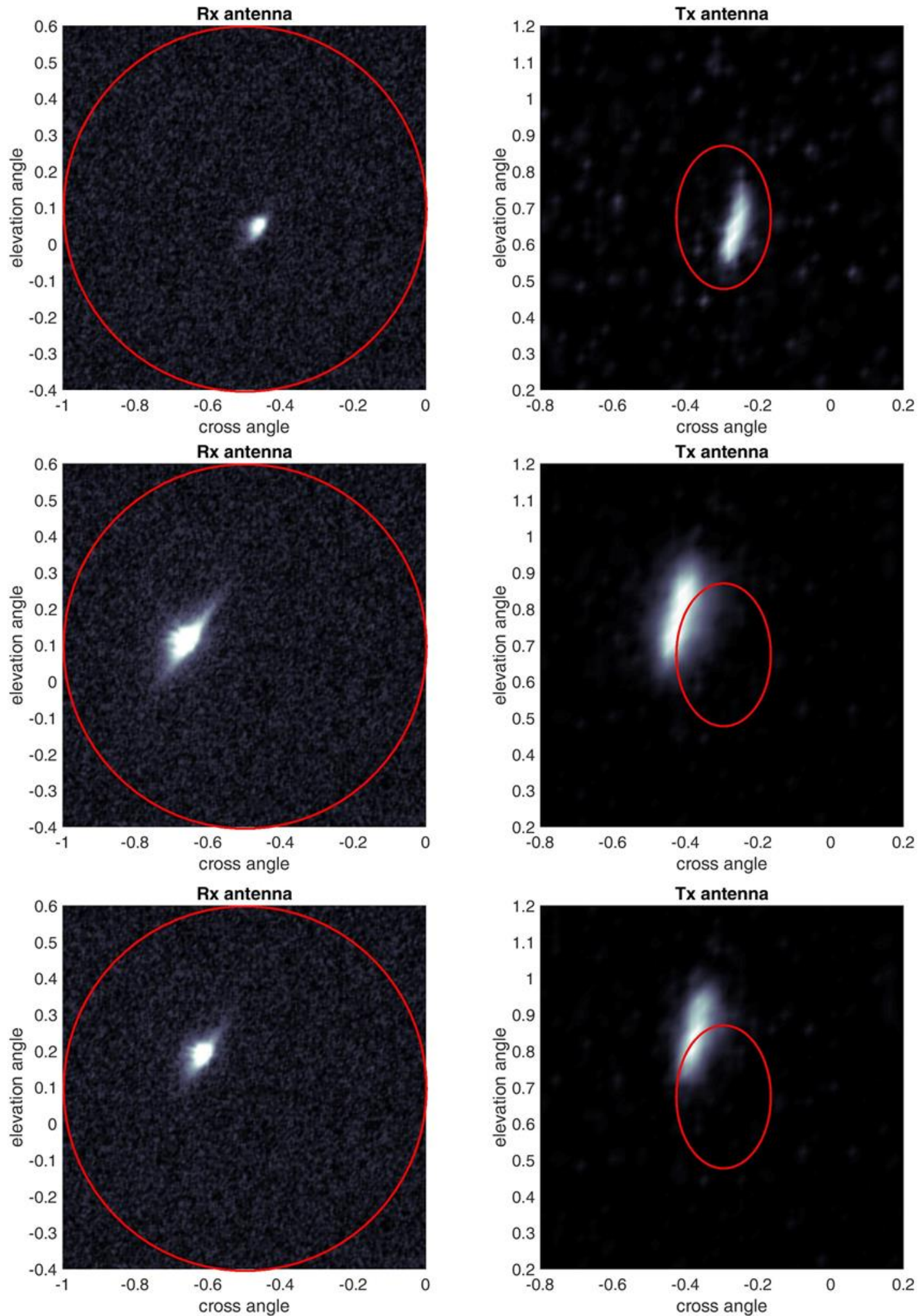

Figure 8. Optical snapshots of the ISS during an orbit path. The red ring indicates the antenna half-power beam width, different for Tx and Rx because of the aperture size. The bright white blot shows the reflected sunlight from the ISS in twilight conditions for three different states. 


\subsection{Data processing}

Beside the challenges in hardware a key part of very high resolution radar for imaging of satellites in space is the processing of the recorded pulses [8]. To get a well-focused ISAR image, a couple of subsequent signal processing steps have to be carried out as indicated in Figure 9. During an orbit path a receive window of $5 \mathrm{~km}$ length is adapted to the distance to the satellite. Hence, the first two steps are the allocation of the pulse with respect to the receive window and perform a basic range correction based on the predicted TLE position. In a second step the effects of the atmosphere is corrected based on the meteorological data and the Total Electron Content TEC value.

An object in the LEO has a velocity of more than $7 \mathrm{~km} / \mathrm{h}$ yielding to a considerable Doppler shift. Assuming the very high bandwidth this shift is also frequency dependent and would cause a defocusing effect in the radar image. So the fourth step is the correction of this frequency dependent Doppler shift in the frequency domain, again based on the predicted TLE position.

After this basic correction the calibration measurements described in 3.2 are calculated and processed to correct the frequency response of the instrument. In a next step the pulse compression is executed leading to the focusing in range direction. After the compression still a range migration can be identified caused by the inaccuracy of the TLE data and by remaining uncertainty in the compensation of atmospheric range delay. In the actual processor the additional mitigation of the residual range error is accomplished by extracting a robust point target in the focused range profiles and performing a range correction for it. In future this can be solved by a dedicated autofocus algorithm. Finally for ISAR processing a backprojection algorithm [4] is applied

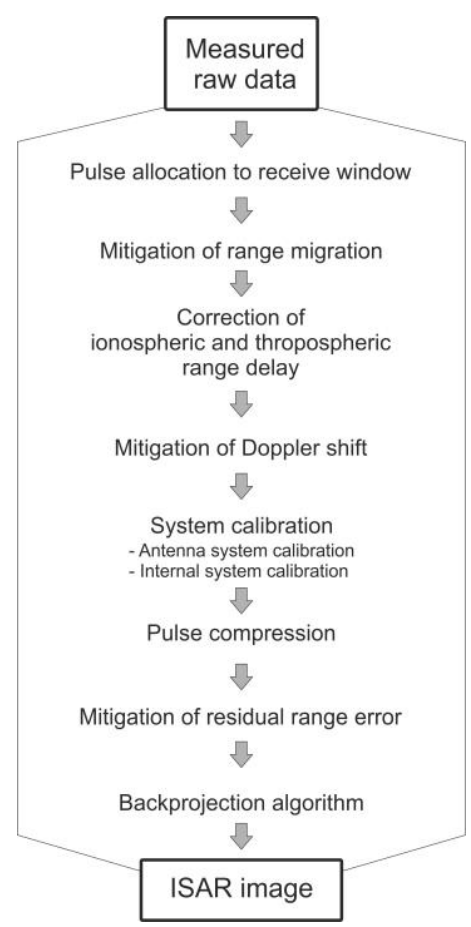

Figure 9. Signal processing steps for the IoSiS basic ISAR processor.

\section{VERY HIGH RESOLUTION ISAR MEASUREMENT RESULT}

The main purpose of IoSiS was the investigation in a technology for observation of objects in space with a centimeter range resolution and to learn from the challenges to design a new operational system with even a higher resolution than IoSiS. By finding solution for the challenges described in chapter 3 and some smaller ones more and solving most of them DLR made a huge step forward. In this paper we can show the first time an image with the nominal resolution of less than $6 \mathrm{~cm}$. The observation object, the international space station ISS, is on the one hand an easy object because of the huge size and RCS value, on the other hand are the available TLE data one of the worst, again because of the huge 
size. The ISAR image of the ISS by processing range profiles of $2.8 \mathrm{GHz}$ bandwidth and using the backprojection algorithm is shown in Figure 10.

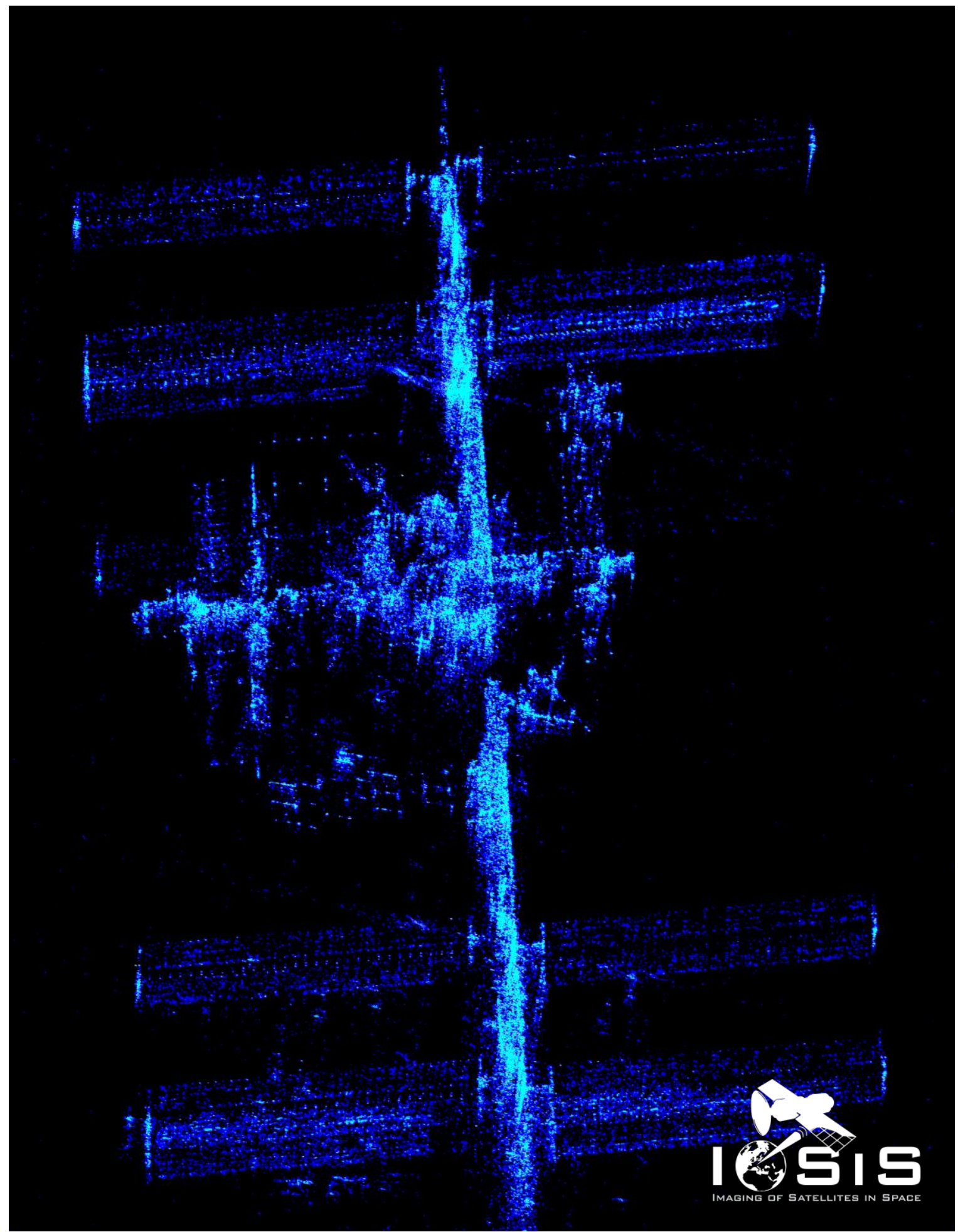

Figure 10. First centimeter-resolution radar image of the international space station ISS acquired by IoSiS. The spatial resolution is about $6 \mathrm{~cm}$ in range direction and $4 \mathrm{~cm}$ in azimuth direction. 
In Figure 10 the observation angle was larger than necessary for squared resolution cells so the azimuth resolution of the image is higher with $4 \mathrm{~cm}$. The number of pixels is about 2000 by 1400 without oversampling. Hence the radar image shows a lot of details and an impressive overview of the whole station. Luckily at the imaging time the solar panels were oriented in a visible direction for an impressive image. In normal orientation of the panels most of the energy is reflected away from the radar receiver. In the center region of the ISS, providing the most complex structure, many details are identifiable like single modules. For example, in the lower right part on the main center structure above the solar panels the Canadarm2 is clearly visible. Also clearly visible is the Kibo's exposed facilities close to the upper right panels. Notable are the exposed facility payloads with a size of $0.8 \mathrm{~m} \times 1.0 \mathrm{~m} \times 1.85 \mathrm{~m}$. These cubes are configurable and the position and quantity can change, visible in the image. With more sophisticated image processing tool using for instance an apodization algorithm, visualizing of many more details is feasible [9].

\section{V. CONCLUSION}

In this paper the challenges in very high resolution imaging of satellites and objects in space was presented based on the experiences of the IoSiS system. The instrument was established by DLR for future high-performance space surveillance radar system, investigating advanced radar concepts for future issues, being not adequately addressable by today's classic imaging approaches. The challenges to generate an image of an object in the LEO with centimeter resolution are enormous. Only if considering all parts of a system including instrument design, proper error correction and calibration, antenna steering unit, and an adapted ISAR processor a focused image is achievable. With the knowledge of the solution of all mentioned challenges, the first time a very high resolution ISAR image with less than $6 \mathrm{~cm}$ resolution of an object in space could be presented. The image of the ISS recorded with IoSiS shows a remarkable granularity enabling a profound interpretation and analyses of the object of interest.

\section{REFERENCES}

[1] Jirousek, M., Iff, S., Anger, S., Peichl, M., "GigaRad - a multi-purpose high-resolution ground-based radar system concept, error correction strategies and performance verification”. International Journal of Microwave and Wireless Technologies, 7(3/4), pp 443-451. Cambidge University Press. (2015)

[2] Anger, S., Peichl, M., Dill, S., Jirousek, M., Schreiber, E., "IoSiS - A high-performance imaging radar for surveillance of objects in low earth orbit", Proceedings of European Conference of Synthetic Aperture Radar (EUSAR), Hamburg, Germany, 2016.

[3] Anger, S, Jirousek, M., Peichl, M. "GigaRad - a versatile high-resolution ground-based pulse radar for advanced remote sensing research”, Proc. EUSAR, Berlin, Germany, 2014.

[4] Mensa, D.: "High Resolution Radar Cross-Section Imaging", Artech House, 1981

[5] Kahle K., Schulze D., Schättler B, „TerraSAR-X/TanDEM-X Mission Operations Segment - Orbit and Attitude Product Specification”, German Space Operations Center (GSOC), Oberpfaffenhofen, February. 2017

[6] Anger S., Jirousek M., Dill S., Schreiber E., Peichl M., „Imaging of satellites in space (IoSiS): challenges in image processing of ground-based high-resolution ISAR data", Proc. SPIE 10633, Radar Sensor Technology XXII, doi: 10.1117/12.2304945, Orlando, 2018

[7] Jirousek M., Anger S., Dill S., Schreiber E., Peichl M., "IoSiS: a radar system for imaging of satellites in space," Proc. SPIE 10188, Radar Sensor Technology XXI, 101880Y (2017); doi: 10.1117/12.2261967

[8] Anger S., Jirousek M., Dill S., Peichl, M. „IoSiS - A high performance experimental imaging radar system for space surveillance" Radar Conference, Boston, USA, (2019).

[9] Kempf, T. ,Peichl, M. , Dill, S. und Süß, H. (2007) 3D Tower-Turntable ISAR Imaging. In: 37th European Microwave Conference 2007. Microwave Journal. 37th European Microwave Conference 2007, 2007-10-08 2007-10-12, München.

[10] UCS Satellite Database , https://www.ucsusa.org/nuclear-weapons/space-weapons/satellitedatabase\#.XDZDs217ksc (2019-03-21)

[11] Royal Air Force (RAF) Fylingdales https://www.raf.mod.uk/our-organisation/stations/raf-fylingdales/ (201903-21) 
[12] Stone, M., Banner, G., " Radars for the Detection and Tracking of Ballistic Missiles, Satellites, and Planets”, Lincoln Labs Journal Vol 12 Nr 2 p 217-244, 2000

[13] J. Ender, L. Leushacke, A. Brenner and H. Wilden, "Radar techniques for space situational awareness," 12th International Radar Symposium (IRS), Leipzig, pp. 21-26 (2011) 